\title{
DESARROLLO DE NUEVOS SERVICIOS EN ENTIDADES FINANCIERAS DE LA CIUDAD DE PIURA, PERÚ
}

\section{DEVELOPMENT OF NEW SERVICES IN FINANCIAL ENTITIES OF THE CITY OF PIURA, PERU}

\section{JENNY CECYL SAGÁSTEGUI HERNÁNDEZ', EDUARDO SÁNCHEZ RUIZ²}

1 Universidad de Piura. jenny.sagastegui@gmail.com

2 Universidad de Piura. eduardo.sanchez@udep.pe

RESUMEN

Se realizó un análisis en entidades financieras de la ciudad de Piura, donde se busca conocer el grado de participación de un cliente antes, durante y después del lanzamiento de un nuevo servicio. Esto responde a la inquietud de muchas empresas de saber cuán importante es considerar al cliente como un miembro más de la organización y ejercer en ella la práctica de desarrollo de nuevos servicios (DNS). Los resultados permiten diseñar un nuevo modelo estándar, que las empresas dedicadas a la prestación de bienes intangibles 0 servicios, particularmente las empresas financieras deben seguir para que el servicio propuesto resulte exitoso y sobretodo responda a lo que el cliente está buscando. Finalmente se habla detalladamente de cada uno de los pasos que forman parte del modelo a seguir, haciendo hincapié de las actividades que una entidad financiera no debe dejar de hacer para poder tener a un cliente satisfecho y pueda sacar ventaja competitiva respecto a la competencia.

PALABRAS CLAVE: desarrollo de servicios, innovación de servicios, nuevos servicios, bienes intangibles.
It was performed an analysis on financial institutions in the city of Piura where is searched to define the grade of participation of a customer before, during and after the release of a new service. This answers to the preoccupation of many companies about knowing how important is to consider the customer as a member of the organization and apply on them the new services development practice. The results allow us to design a new standard model, that companies working with intangible goods or services, particularly financial companies, should follow to be successful in the proposal of those services and, most of all, to respond to customer needs. Finally, each step that take part of the model is presented in detail, emphasizing on the activities that a financial institution should not put aside in order to satisfy the customer and get a competitive advantage over the competition.

KEYWORDS: service development, service innovation, new services, intangible goods. 
INTRODUCCIÓN

La intensa competencia, los cambios tecnológicos y la economía creciente han hecho que el Desarrollo de Nuevos Servicios (DNS) sea la clave del éxito de muchas compañías, por lo que éstas se ven en la necesidad de innovar constantemente para ir al ritmo de cambio.

El crítico rol de la innovación ha sido reconocido en los bienes físicos; sin embargo el desarrollo de nuevos servicios no recibe tanta atención por parte de los directivos de las empresas enfocadas en los servicios. (Edvarson, 2000)

Los servicios proporcionan ayuda, utilidad, experiencia, información u otro contenido intelectual y, la mayoría del valor es intangible en lugar de residir en cualquier producto físico (Department of Industry, 1999). El DNS involucra un trato más cercano con el cliente, una cadena completa que involucra desde la planificación hasta la entrega del servicio. El valor final del servicio es percibido por el cliente.

\section{METODOLOGÍA INNOVACIÓN DE SERVICIOS}

Innovar en servicios es crear o modificar un servicio e introducirlo en el mercado. También consiste en la conversión de ideas en productos, procesos o servicios. Estas ideas pueden ser tecnológicas, comerciales y organizacionales que involucran procesos nuevos o mejorados y que implican nuevas formas de organización y gestión de la empresa o nuevos comportamientos en el mercado.

DESARROLLO DE NUEVOS SERVICIOS (DNS)

Debido a las nuevas tendencias en el mercado tales como el aumento de las expectativas de los clientes, y avances en la tecnología, se ha incrementado la competencia en el mercado, razón por la cual las empresas se ven en la necesidad de desarrollar nuevos servicios. Sin embargo, se observa que los servicios son desarrollados sin una focalización de sus objetivos estratégicos, ni las competencias necesarias. (Martin y Home, 1993) (Kelly \& Storey, 2000). Esto constituye ser el origen de un alto porcentaje de fracaso en los nuevos servicios, causado por la falta de un eficiente proceso de desarrollo.

El DNS asigna tareas y provee de objetivos a las compañías de servicio y a sus directivos (Barzack, 1995). Estos objetivos involucran a decidir cómo se deben organizar para el desarrollo de un nuevo servicio y cómo desarrollarlos de tal manera que respondan a las necesidades del cliente.
MODELO DEL PROCESO DE DNS

En la actualidad para crear nuevos servicios con una tasa más elevada de innovación, las empresas primero deben llegar a un mayor nivel de compresión de la importancia de la formalización del proceso de DNS. Entre los modelos de estudio se encuentran: modelo de desarrollo secuencial o también conocido como Modelo Stage Gate, modelo cíclico del proceso de DNS, modelo basado en factores organizacionales, modelo orientado a los clientes, y modelo de desarrollo de la experiencia.

De todos los modelos mencionados, el modelo en el cual se centra la investigación es el que se encuentra orientado a los clientes. Este modelo fue propuesto por Alam y Perry, y particularmente, encuentra su aplicación en el sector de servicios financieros, pero puede ser utilizado en otros sectores, efectuando algunos cambios.

En la figura 1, puede apreciarse el modelo orientado a los clientes, y una variación del mismo.

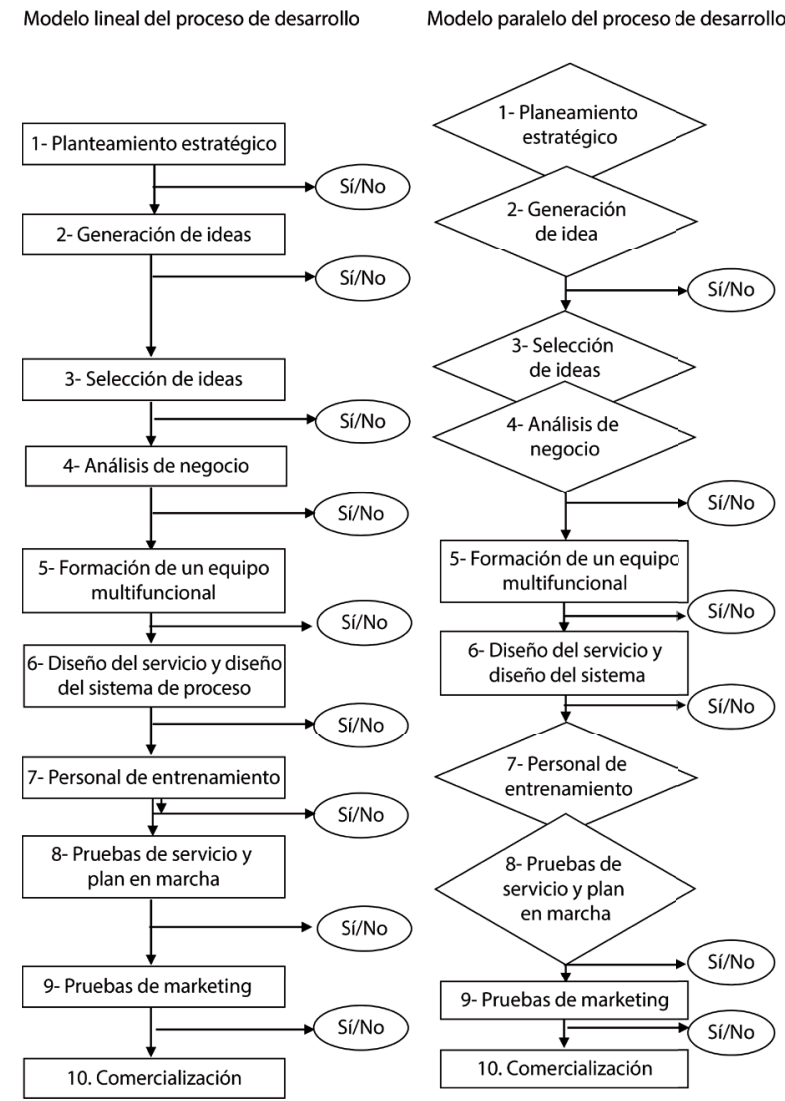

Figura 1. Dos modelos de DNS Fuente: Alam y Perry, 2002. 
Este modelo es el resultado de una investigación empírica en las empresas que han puesto de manifiesto la forma en que se viene desarrollando el proceso de DNS.

Según investigaciones se afirma que hay dos variantes de este modelo: la presencia de fases que se desarrollan en paralelo o en secuencia, y esto es en función de la dimensión de la empresa. Tres pares de etapas pueden llevarse a cabo simultáneamente: planificación estratégica y generación de la idea; selección de idea y análisis de negocio; y entrenamiento del personal y servicio de pruebas con el fin de obtener una mayor flexibilidad. Se ha dicho ya en anteriores investigaciones que los servicios tienden a involucrar más a menudo y con mayor intensidad al cliente (Harris, 2001; Alam, 2002; Martin, 1999), por lo que se puede afirmar que la orientación al cliente debe tener un papel fundamental en el proceso de DNS.

\section{MERCADO FINANCIERO}

El sistema financiero en el Perú, está conformado por el conjunto de instituciones bancarias, financieras y demás empresas e instituciones de derecho público o privado, debidamente autorizadas por la Superintendencia de Banca, Seguro y AFP, que operan en la intermediación financiera (actividad habitual desarrollada por empresas e instituciones autorizadas a captar fondos del público y colocarlo en forma de créditos e inversiones).

El banco financiero se clasifica en:

- Sistema financiero bancario. Constituido por el conjunto de instituciones bancarias del país. Se encuentra el Banco Central de Reserva del Perú (BCRP), Banco de la Nación y banca comercial (Banco de Crédito del Perú, Banco Continental, etc.).

- Sistema financiero no bancario. Constituido por financieras, cajas municipales de ahorro y crédito (orientadas preferentemente a las pequeñas y $\mathrm{mi}^{-}$ cro-empresas), entidades de desarrollo a la pequeña y microempresa (Edpyme), caja municipal de crédito popular, cajas rurales (otorgan financiamiento preferentemente a los empresarios de la pequeña y micro-empresa).

- Empresas especializadas. Instituciones financieras, que operan como agente de transferencia y registros de las operaciones o transacciones del ámbito comercial y financiero. Dentro de este rubro se encuentran las empresas de arrendamiento financiero, empresas de factoring, empresas afianzadoras y de garantías, y empresas de servicios fiduciarios.

En el Perú, en los últimos años, las empresas financieras mantuvieron una estrategia agresiva dirigida a la banca minorista. De esta manera se observó un considerable incremento en diversos créditos: más de $10 \%$ en el crédito de consumo; un incremento de $22,7 \%$ en créditos hipotecarios y los créditos a microempresas, aumentaron en $32 \%$. Este crecimiento fue originado por la continua expansión del consumo privado, la mejora en la capacidad de pago de la población, y la mayor competencia en el sistema, la cual derivó en menores tasas activas.

Al analizar la situación del sistema financiero en los últimos años, se observa el creciente dinamismo de las instituciones financieras no bancarias, lo que indicaría que ahora los clientes tienen más alternativas y, por lo tanto, existe un poco más de competencia en el sector. No obstante las limitaciones económicas, administrativas y logísticas de las cajas y edpymes, vienen creciendo en el mercado financiero, logrando posicionarse en ciertos segmentos, especialmente en las provincias y en actividades que no interesan mayormente a la gran banca comercial, como la agricultura.

\section{PROCESOS DE INNOVACIÓN}

Con la finalidad de analizar el enfoque de desarrollo de los servicios en el mercado financiero se realizaron entrevistas a directivos y gerentes de diversas empresas del sector.

En la Tabla 1, se muestra s los diversos servicios ofrecidos por las entidades financieras.

\section{PROCESAMIENTO DE INFORMACIÓN}

De acuerdo a las entrevistas realizadas, se efectuó el procesamiento de la información obtenida comparando los procesos de innovación con el modelo de desarrollo de nuevos servicios propuesto por Alam y Perry (2002).

En las respuestas obtenidas (Tabla 2), se manifiesta que las entidades financieras ponen en prácticas diversas etapas en el desarrollo de sus servicios como se muestra en el cuadro resumen. Se visualiza que las etapas generación de idea y comercialización son llevadas a cabo en todas las organizaciones (el total de columnas de la tabla). Estas dos etapas se encuentran entre las más importantes ya que ambas tienen 
TABLA 1. PROCESOS DE INNOVACIÓN REALIZADOS EN LAS ENTIDADES FINANCIERAS

\begin{tabular}{|c|c|c|}
\hline $\begin{array}{l}\text { ENTIDAD } \\
\text { FINANCIERA }\end{array}$ & $\begin{array}{l}\text { PROCESO DE } \\
\text { INNOVACIÓN }\end{array}$ & DESCRIPCIÓN \\
\hline \multirow{3}{*}{$\mathrm{BCP}$} & Agente $\mathrm{BCP}$ & $\begin{array}{l}\text { Se entiende por cajeros corresponsales, según las normas de la SBS (Superintendencia de Banca, Seguros y AFP), como operado- } \\
\text { res de servicios constituidos por personas naturales o jurídicas que funcionan en establecimientos propios o de terceros, distintos } \\
\text { de los del sistema financiero respecto de los cuales existen acuerdos contractuales para prestar, a través de dichos operadores de } \\
\text { servicios y bajo responsabilidad de la empresa del sistema financiero, las operaciones y servicios autorizados que ésta determine. }\end{array}$ \\
\hline & $\begin{array}{l}\text { Cuenta cero } \\
\text { y cuenta } \\
\text { libre }\end{array}$ & $\begin{array}{l}\text { Son cuentas de ahorro dirigidas hacia el segmento personas, cuya diferencia radica según la preferencia del cliente, respecto al } \\
\text { número de operaciones que se desee realizar ya sea por ventanilla o cajero. La cuenta cero tiene un monto mínimo de ahorro, en } \\
\text { cambio la cuenta libre no tiene, por eso la cuenta libre tiene más ventajas, en cuanto al número de operaciones libres sin comisión. }\end{array}$ \\
\hline & $\begin{array}{l}\text { Cuenta } \\
\text { sueldo }\end{array}$ & $\begin{array}{l}\text { La cuenta sueldo es una cuenta de ahorros en la que se registran, los abonos derivados de las remuneraciones del cliente. } \\
\text { El objetivo primordial de este servicio es la bancarización, que significa utilizar intensivamente al sistema financiero para facilitar } \\
\text { las transacciones efectuadas entre los agentes económicos. } \\
\text { - La bancarización posibilita la monetización de la economía y es condición necesaria para salir de la pobreza. } \\
\text { - Una mayor bancarización es el resultado de mayor estabilidad de los sistemas financieros y confianza en éstos. }\end{array}$ \\
\hline $\begin{array}{l}\text { ENTIDAD } \\
\text { FINANCIERA }\end{array}$ & $\begin{array}{l}\text { PROCESO DE } \\
\text { INNOVACIÓN }\end{array}$ & DESCRIPCIÓN \\
\hline BBVA & $\begin{array}{l}\text { Tarjeta Blue } \\
\text { joven }\end{array}$ & $\begin{array}{l}\text { Blue joven es un programa de BBVA, de acuerdo con los intereses y necesidades de los jóvenes entre } 15-25 \text { años, a través del cual } \\
\text { se accede a productos y servicios financieros en base a ofertas de música, cine, informática, viajes, etc. Le da amplio límites de } \\
\text { crédito para un carro, departamento, entradas a discotecas, etc. }\end{array}$ \\
\hline \multirow{3}{*}{$\begin{array}{l}\text { Caja } \\
\text { Municipal } \\
\text { Sullana }\end{array}$} & $\begin{array}{l}\text { Crédito } \\
\text { agrícola }\end{array}$ & $\begin{array}{l}\text { Servicio dirigido a productores agropecuarios para financiar actividades económicas productivas. Este servicio es clasificado } \\
\text { según su finalidad y plazo: } \\
\text { - De sostenimiento, para financiar actividades productivas, cuyo reembolso es a corto plazo. } \\
\text { - De capitalización y comercialización, para financiar la adquisición de bienes de activo fijo y con reembolso a mediano plazo. } \\
\text { Su tecnología financiera le permite evaluar en el terreno los sectores que reúnen las mejores condiciones agrícolas (suelo, agua, } \\
\text { drenaje), menor riesgo de inundación, rendimientos por cultivo, niveles de tecnología utilizados, entre otros aspectos. }\end{array}$ \\
\hline & Caja Max & $\begin{array}{l}\text { Un cajero corresponsal es un punto de atención autorizado por la SBS que se ubica en un establecimiento comercial distinto del } \\
\text { Sistema Financiero, con el cual la CMAC-Sullana mantiene un acuerdo contractual y a través de ellos realizar las operaciones y } \\
\text { servicios autorizados. }\end{array}$ \\
\hline & Compu plan & $\begin{array}{l}\text { El crédito Compu plan está dirigido a las pymes, profesionales y estudiantes de todo el país que quiera obtener computadoras. } \\
\text { Para acceder a este plan no se requiere cuota inicial y el crédito es en moneda nacional. }\end{array}$ \\
\hline $\begin{array}{l}\text { Banco del } \\
\text { Trabajo }\end{array}$ & $\begin{array}{l}\text { Crédito } \\
\text { jubilado } 85\end{array}$ & Es un crédito ofrecido a personas mayores de 85 años y a un plazo máximo de 12 meses. \\
\hline
\end{tabular}

Fuente: Elaborado a partir de las entrevistas realizadas por los autores.

puntaje 10, y porque la generación de ideas, es el punto de partida de un proyecto y la comercialización es la última etapa que constituye la línea de meta del proyecto.

Así mismo se consideran importantes el planeamiento estratégico, entrenamiento de personal, formación de grupo multidisciplinarios, diseño de servicio y sistema del proceso. En contraste, las pruebas piloto y pruebas de marketing, son actividades consideradas como menos importantes porque las entidades financieras tienden a hacer caso omiso de estas etapas con el fin de acelerar el proceso general de desarrollo del servicio y es como lo manifiestan muchos directivos que por razones de urgencia, se omiten las pruebas piloto y plan de marketing y saltan directamente al lanzamiento del servicio.

Además se puede concluir que la mayoría de las entidades financieras muestran actitud reactiva, porque responden a los servicios y cambios del mercado pero solo cuando están presionados por el medio. Incluso sus servicios son imitación de los brindados por otras entidades financieras, en lugar de ser ellos mismos innovadores. Así, ellos simplifican la realización de estudios de mercado y lanzan el servicio sin desarrollar una metodología estructurada, ni formal. Esto, a su vez refleja que la etapa de selección de ideas es poco practicada en las entidades financieras.

Podemos afirmar debido a todo lo mencionado anteriormente que el problema de las entidades financieras se encuentra en la deficiencia en el diseño del nuevo servicio por la falta participación del cliente, dado que lanzan un servicio sin saber exactamente si llegará a satisfacer la necesidad de éstos, limitándose a aplicar lo que la competencia normalmente hace. 
TABLA 2. CUADRO RESUMEN DE ANÁLISIS DE INFORMACIÓN DE DESARROLLO DE SERVICIOS DE LAS ENTIDADES FINANCIERAS ENTREVISTADAS

\begin{tabular}{|c|c|c|c|c|c|c|c|c|c|c|c|}
\hline \multirow{2}{*}{$\begin{array}{l}\text { ENTIDADES } \\
\text { Servicios }\end{array}$} & \multicolumn{3}{|c|}{ BCP } & \multirow{2}{*}{$\begin{array}{c}\text { BBVA } \\
\text { Tarjeta } \\
\text { Blue joven }\end{array}$} & \multicolumn{4}{|c|}{ CMAC SULLANA } & \multirow{2}{*}{$\begin{array}{c}\text { BANCO DEL } \\
\text { TRABAJO } \\
\text { Crédito } \\
\text { jubilado } 85\end{array}$} & \multirow{2}{*}{$\begin{array}{l}\text { SCOTIABANK } \\
\text { Tarjeta única }\end{array}$} & \multirow[t]{2}{*}{ TOTAL } \\
\hline & Agente & $\begin{array}{l}\text { Cuenta } \\
\text { cero y libre }\end{array}$ & $\begin{array}{l}\text { Cuenta } \\
\text { sueldo }\end{array}$ & & $\begin{array}{l}\text { Crédito } \\
\text { prendario }\end{array}$ & $\begin{array}{l}\text { Crédito } \\
\text { agrícola }\end{array}$ & $\begin{array}{l}\text { Caja } \\
\text { máx. }\end{array}$ & $\begin{array}{l}\text { Compu } \\
\text { plan }\end{array}$ & & & \\
\hline \multicolumn{12}{|l|}{ Etapas } \\
\hline a) Planeamiento estratégico & $x$ & $x$ & $x$ & $x$ & $x$ & $x$ & $X$ & $x$ & $x$ & $x$ & 10 \\
\hline b) Generación de Ideas & $x$ & $x$ & $x$ & $x$ & $x$ & $x$ & $x$ & $x$ & $x$ & $x$ & 10 \\
\hline c) Selección de Ideas & & $x$ & & & & & & & $x$ & & 2 \\
\hline d) Análisis de Negocio & $x$ & $x$ & $x$ & $x$ & $x$ & $x$ & $x$ & $x$ & $x$ & $x$ & 10 \\
\hline $\begin{array}{l}\text { e) Formación de grupos } \\
\text { multidisciplinarios }\end{array}$ & $x$ & $x$ & $x$ & $x$ & $x$ & $x$ & $x$ & $x$ & $x$ & $x$ & 10 \\
\hline $\begin{array}{l}\text { f) Diseño del servicio y } \\
\text { diseño del proceso }\end{array}$ & $x$ & $x$ & $x$ & $x$ & $x$ & $x$ & $x$ & $x$ & $x$ & $x$ & 10 \\
\hline $\begin{array}{l}\text { g) Entrenamiento del } \\
\text { personal }\end{array}$ & $x$ & $x$ & $x$ & $x$ & $x$ & $x$ & $x$ & $x$ & $x$ & $x$ & 10 \\
\hline $\begin{array}{l}\text { h) Pruebas de servicio y } \\
\text { plan en marcha }\end{array}$ & $x$ & & & & & & & & & & 1 \\
\hline i) Pruebas de marketing & & & & & & & & & & & 0 \\
\hline j) Comercialización & $x$ & $x$ & $x$ & $x$ & $x$ & $x$ & $x$ & $x$ & $x$ & $x$ & 10 \\
\hline Total & 8 & 8 & 8 & 7 & 7 & 7 & 7 & 7 & 8 & 7 & \\
\hline
\end{tabular}

Fuente: Elaboración propia.

\section{RESULTADOS Y DISCUSIÓN} MODELO PROPUESTO

Después de todo lo analizado, se considera esencial medir la calidad del servicio brindado al cliente como un elemento imprescindible en el desarrollo de un nuevo servicio. También es importante la necesidad de la participación del cliente, por eso a continuación se propone un modelo de desarrollo de nuevos servicios para el mercado financiero peruano que involucra la participación del cliente en las diversas etapas de un proceso de DNS.

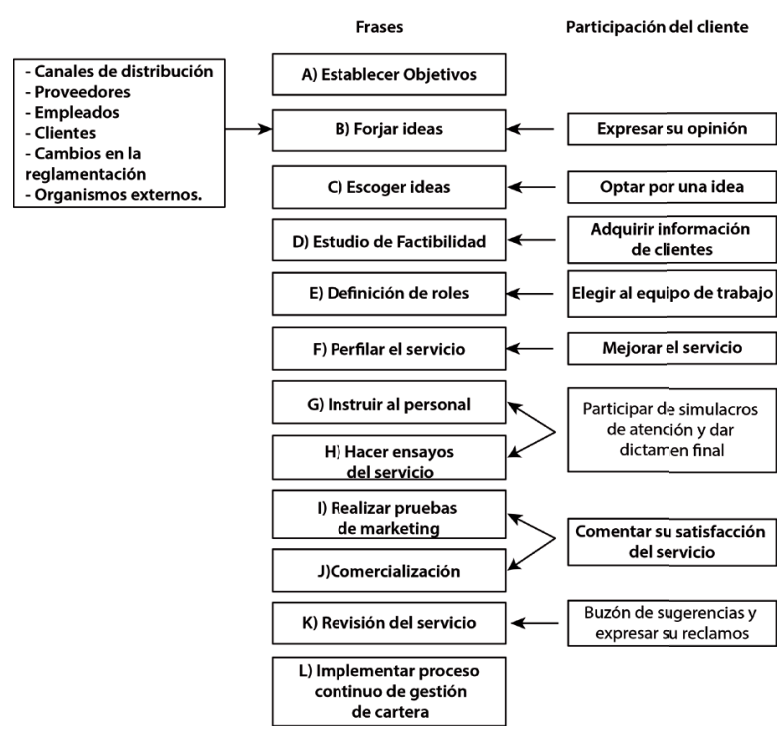

Figura 2. Modelo Propuesto

\section{FASES DEL DNS}

A. ESTABLECER OBJETIVOS

Se debe establecer la necesidad de planificación, definiendo el escenario estratégico de la innovación, los objetivos de la actividad de nuevos servicios y el programa para lograr esos objetivos. Las diferentes opciones de estrategias comerciales pueden traducirse en el desarrollo de nuevos planes de servicio. En esta fase se debe establecer los objetivos del nuevo servicio y analizar si se alinean con los de la entidad financiera. Luego se debe definir la trayectoria del proceso de innovación en términos de objetivos del cliente y la labor que la entidad financiera debe realizar para lograr esos objetivos, asimismo se debe definir las actividades para lograr los objetivos del servicio.

B. FORJAR IDEAS

Las ideas de nuevos servicios pueden ser generadas de muchas maneras, éstas pueden surgir dentro y fuera de las entidades financieras, o ser el resultado de formales o informales procedimientos de búsqueda. El éxito de las empresas radica en establecer sistemas y procedimientos para estimular la generación de ideas a largo plazo.

Dado que las ideas de nuevos servicios pueden surgir en cualquier lugar, los empleados han sido considerados durante mucho tiempo como una fuente importante de ganar nuevas 
ideas de servicios, y esto se debe a la proximidad de los empleados con los clientes dado que los empleados pueden recoger las inquietudes de los clientes y las pueden transmitir a la empresa para dar soluciones alternativas frente a cualquier inconveniente.

Cada servicio nuevo es resultado de una idea, y cuanto más numerosas sean las ideas generadas, más probabilidades habrán de seleccionar la mejor.

\section{SELECCIÓN DE IDEAS}

El objetivo de la selección de nuevas ideas significa asignar recursos entre los proyectos de DNS que tienen más probabilidad de ayudar a la entidad financiera a cumplir con sus objetivos. Este proceso puede ser una sola o una serie de actividades de múltiples etapas.

Los criterios de selección deben centrarse en factores tales como las ventajas del nuevo servicio y la superioridad, las ventajas económicas para el usuario final y el crecimiento del mercado que está siendo blanco de ataques. Es importante destacar que la selección debe ser un proceso continuo durante todo el desarrollo de servicios y no sólo una discreta decisión en un punto clave en el proceso. Es por esta razón que se considera que las etapas de selección y evaluación deben realizarse de manera simultánea.

Para una correcta selección de ideas, se debe realizar la clasificación de las diversas propuestas por orden de categorías, eligiendo el conjunto más atractivo posible, dentro de los recursos de la empresa. Debe procurarse no caer en dos tipos de errores: omisión, desechando una idea que podría ser útil, y comisión, desarrollando y comercializando una idea que no vale la pena.

\section{ESTUDIO DE FACTIBILIDAD}

Esta etapa está relacionada con analizar la capacidad de sus recursos disponibles, ya que cuando se trata de comprometer cierto número de iniciativas, no conviene abarcar más de lo que se puede.

Lo primero que se debe realizar es la definición y análisis de las especificaciones, debido a que son necesarios para satisfacer las necesidades de los clientes. Las especificaciones son las representaciones de las necesidades en forma de diagramas y documentos de texto estructurado que se elaboran en relación de los diversos requisitos de los componentes.

El análisis de requisitos es una parte importante de la solución de negocio, que abarca las actividades que participan en el examen de la información acerca de la necesidad comercial y el alcance de una nueva o modificada solución de negocio; las necesidades de información se descomponen hasta que las especificaciones de los requisitos sean precisas, sin ambigüedades, y completas.

Para hacer el estudio de factibilidad, es necesario que las entidades financieras conozcan con profunda claridad sobre su competencia y su entorno empresarial. Además resulta fundamental que adquieran información sobre sus clientes. Para esto, deben recopilar datos y emplear técnicas para el análisis de su data, luego deben realizar un análisis final para poder orientar su proceso de análisis, incluyendo la definición de sus problemas. Las entidades financieras deben realizar una evaluación realista de las fortalezas, debilidades, la viabilidad, y el valor de su negocio.

\section{E. DEFINICIÓN DE ROLES}

El siguiente paso es definir las funciones específicas tanto para el equipo de la entidad financiera como un grupo de clientes. Los roles del personal de la entidad financiera se definen para que el cliente entienda la serie de habilidades y actividades que cada miembro del equipo debe tener. Los roles del cliente son definidos para informar al cliente del compromiso del proyecto liderado de habilidades y los niveles de experiencia necesarios para que el DNS tenga éxito. Estos equipos deben estar formados por personas con diferentes conocimientos y habilidades funcionales de trabajo, todos dirigidos hacia un objetivo común. Se puede incluir a empleados de todos los niveles de una organización aunque los miembros también pueden venir de fuera de una organización (proveedores, clientes clave o consultores).

Se debe optar por la multidisciplinariedad como criterio de formación de los equipos de trabajo, es decir para el desarrollo de un nuevo servicio deben intervenir las áreas de sistemas, marketing, ventas, etc.

\section{F. PERFILAR EL SERVICIO (BOSQUEJO DEL SERVICIO)}

Para delinear un servicio, se requiere de métodos y herramientas para el control de nuevos elementos de este proceso, tales como el tiempo y la interacción de los actores del servicio. Se propone tres direcciones principales:

- Identificación de los actores involucrados en la definición del servicio, utilizando los instrumentos analíticos. 
- Definición de los posibles escenarios del servicio, verificación de casos de uso, secuencia de acciones y el papel de los actores, con el fin de definir los requisitos para el servicio, la lógica y su estructura organizativa.

- Representación del servicio, utilizando técnicas que ilustren todos los componentes del servicio, incluyendo elementos físicos, interacciones, vínculos lógicos y secuencias temporales.

Es recomendable utilizar la técnica de los storyboards (ilustraciones mostrada en secuencia conelobjetivodeservirdeguía para entenderuna historia, previsualizar una animación o seguir la estructura de una película antes de realizarse o filmarse), con el fin de mostrar la interacción en la oficina de la entidad financiera. Las empresas orientadas hacia la innovación tienen que desarrollar una relación de vanguardia con los clientes. En síntesis el papel del cliente en la innovación del servicio debe ser contribuir con conocimientos, experiencias, expresar sus necesidades, expectativas, y su disposición a experimentar y aprender.

\section{G. INSTRUIR AL PERSONAL DE TRABAJO}

La capacitación del personal que interactuará con elcliente es una fase muy importante porque influye en la imagen que el cliente se llevará de la entidad financiera. Para que esta fase sea exitosa, las entidades financieras deben designar una muestra de clientes para que participe en el proyecto de DNS, y, basados en esta muestra, las actividades desarrolladas en el proceso de innovación y su experiencia en las actividades que formarían parte del nuevo servicio, podrían determinar el tipo de entrenamiento requerido.

Es necesario que éste entrenamiento sea brindado por las personas adecuadas, en el momento oportuno, en el nivel correcto de detalle para aprovechar al máximo la productividad del cliente en el proceso.

H. PROBAR EL SERVICIO En esta etapa, se ensaya por primera vez el servicio en ambientes reducidos pero bien seleccionados, con la información que pretendería representar las reacciones del consumidor. La entidad podría diseñar una encuesta en línea y depositarla en un sitio web protegido con contraseña y el cliente pueda iniciar sesión cuando le resulte conveniente, evaluar el servicio y enviar comentarios, así participaría en una simulación de los procesos de prestación de servicios para sugerir mejoras.
Es importante considerar las siguientes preguntas: ¿cuántas ciudades o zonas deben considerarse en la prueba?, ¿qué ciudades se tomarán en cuenta?, ¿qué duración debe tener la prueba?, ¿qué tipo de información debe obtenerse?, ¿qué se hará con la información recopilada?

I. ENSAYOS DE MARKETING

Es de vital importancia realizar pruebas de un servicio atractivo para el mercado financiero antes de gastar dinero en el lanzamiento y en una campaña publicitaria que podría resultar un total fracaso. Las pruebas de marketing deben llevarse a cabo para ofrecer un ensayo general antes del lanzamiento de un nuevo servicio en una entidad financiera. Estas pruebas proporcionan información para mejorar el desempeño de la entidad y de esta manera poder evitar lamentaciones y gastos innecesarios.

Es imprescindible involucrar al cliente en estas pruebas, permitiéndole que formule comentarios sobre el plan de marketing, comentarios detallados acerca de su satisfacción; también se debe lograr debatir con los clientes, incluso tomar en cuenta sus puntos de vista con el fin de que pueda sugerir mejoras deseadas.

\section{J. COMERCIALIZACIÓN}

Para conseguir entrar en el mercado se debe poner en practica diferentes técnicas de comercialización, por ejemplo: publicidad en masa, la distribución y las técnicas de branding (consiste en basarse en el poder de la marca como elemento diferenciador). Una vez que el mercado se ha asentado y los clientes conocen bien el servicio, el enfoque de la comercialización cambia, las entidades financieras deben tratar de conocer los gustos de sus clientes para adaptar los servicios a sus necesidades y diversificar la producción. Las entidades financieras deberían plantearse las siguientes preguntas:

¿Quién es mi público objetivo? ¿qué características tiene el segmento al que me dirijo?, ¿qué medio es el que más consume (boletín, folleto, volante)?, ¿en qué etapa me encuentro (lanzamiento, promoción, mantenimiento)?, ¿cuánto durará mi campaña?, ¿qué objetivos de ventas tengo?, ¿cuántos clientes quiero captar?, y lo más importante: ¿cuántos clientes captaré a partir de mis acciones?

La mejor promoción la tiene el mejor servicio, como si se tratara de un mandamiento: nombrar un servicio con el apellido paterno, escribir un eslogan genérico, inundar la vía pú- 
blica con volantes, pintar carteles con la firma del servicio. Como Philip Kotler expresó: "Nadie puede ofrecer o vender aquello que nadie va a comprar». En la actualidad, la categoría banca ha reemplazado los mensajes conservadores por un instrumento más innovador: la creatividad.

\section{K. REVISIÓN DEL SERVICIO}

Las entidades financieras deben desarrollar sistemas de seguimiento para vigilar si el servicio cumple con las expectativas de comercialización y por consiguiente puedan reunir información sobre las posibles modificaciones del servicio. Por lo general, es el cliente descontento el que hace oír más su voz. La solución es el comercio en línea, mediante encuestas y cuestionarios asequibles que comprueben la satisfacción del cliente y que soliciten información de contacto. El análisis de éstos resultados permitirá saber el punto de vista preferente de los clientes, diagnosticar algún problema y mejorar así el rendimiento de la entidad financiera. También se podría proponer programas de sugerencias, para recoger las percepciones del cliente sobre sus servicios y ofrecer incentivos de tal manera que se motive al cliente a ser jurado calificador de los mismos servicios.

\section{IMPLEMENTAR PROCESO CONTINUO DE GESTIÓN DE CARTERA} DE PROYECTOS DE DNS

Las entidades deberán implementar un proceso continuo de gestión de cartera de proyectos de DNS. La entidad debe designar un equipo encargado de establecer procesos para la revisión y actualización continua de su cartera de proyectos de DNS, el cual debería incorporar la gestión nacional de la cartera de iniciativas con las siguientes medidas:

- Agregar la cartera de revisión de iniciativas en la orden del día de las reuniones mensuales de personal.

- Programar una reunión de medio día cada seis meses para hacer una revisión exhaustiva de la cartera.

- Establecer un proceso para las nuevas iniciativas.

\section{CONCLUSIONES}

Las entidades financieras no cuentan con un modelo formal en el desarrollo de sus servicios financieros, solo tienden a imitar los servicios de la competencia. Un modelo formal, como el propuesto en este artículo, permitiría realizar un proceso más estructurado y organizado de desarrollo de nuevos servicios.
Un servicio aparentemente simple, es un proceso altamente complejo, que involucra muchas operaciones, experiencias, resultados y percepciones de los clientes. Las compañías que se mueven demasiado rápido, a menudo experimentan problemas tales como el pobre concepto de servicio, un proceso de diseño al azar, la insuficiencia de pruebas y poquísima planificación para un efectivo lanzamiento en el mercado.

Las entidades financieras deberían reconocer que se deben a sus clientes, que son el activo más valioso de su empresa y que sobreviven solo en el caso que haya clientes y crecen mientras mantengan una cartera y puedan captar nuevos clientes. Por lo tanto su entidad debe ser estructurada y gestionada en base a ellos. Es más, resulta imprescindible adoptar una estrategia proactiva, no reactiva, anticipándose a los movimientos del mercado, buscando ser siempre pioneros. Les resultaría más práctico trabajar bajo una teoría de escenarios: no viendo sólo lo que quieren ver, sino anticipándose a los escenarios que se puedan dar. Esto va de la mano a que cuenten con gente calificada y capacitada que escuche a sus clientes e involucrarlos en el desarrollo de sus procesos tal como se explica en la metodología propuesta.

Por último, la metodología propuesta pone énfasis en que las entidades financieras deben contar con un proceso de desarrollo de nuevos servicios orientado al cliente. Esto permite lograr innovación superior y un mayor éxito. La filosofía de la entidad debe ser escuchar al cliente; es más, tener una cultura enfocada en el cliente, la cual debe estar centrada en tres pilares: tratar bien a sus clientes, cumplir las promesas, y ser accesibles.

\section{REFERENCIAS BIBLIOGRÁFICAS}

Alam, I \& Perry, C. (2002) Acustomer-oriented new service development process. Journal of Services Marketing, 16 (6), 515-534.

Edvarsson, B., Gustafsson, A., Kristensson, P. \& Mangnusson, P. (2006). Involving Customers in New Service Development.

Kelly, D. \& Storey, C. (2000). New service development: Initiation Strategies. Journal of Service Industry Managemente, 11(1) , 45-63.

Matthing, J, Sandén, B. \& Edvarson, B.(2004). New service development: Learning from and with customers. International Journal of Service Industry Management, 15 (5), 479-498.

Sánchez Eduardo (2006) New Service Development and Six Sgma-an explorativestudyin theluxurious fivestartshotels in Milan. 